\title{
MRI Changes of the Spinal Subdural Space after Lumbar Spine Surgeries: Report of Two Cases
}

\author{
Toshinori Sakai ${ }^{1,2}$, Koichi Sairyo ${ }^{3}$, Nitin N. Bhatia², Ryo Miyagi', \\ Tatsuya Tamura ${ }^{1}$, Shinsuke Katoh ${ }^{1}$, Natsuo Yasui ${ }^{1}$ \\ 'Department of Orthopedics, Institute of Health Biosciences, \\ The University of Tokushima Graduate School, Tokushima, Japan \\ ${ }^{2}$ Department of Orthopaedic Surgery, University of California, School of Medicine, Irvine, CA, USA \\ ${ }^{3}$ Department of Orthopedic Surgery, Mizonokuchi Hospital, \\ University of Teikyo School of Medicine, Kawasaki, Japan
}

Although magnetic resonance imaging (MRI) is frequently used to assess the lumbar spine, there are few reports in the medical literature that have evaluated using MRI immediately following spinal surgery. Furthermore, descriptions of the subdural changes after lumbar spine surgery are also infrequent. In this paper, we present two cases with subdural change seen on MRI immediately after lumbar surgery. Both the patients had mild symptoms that resolved spontaneously, and the follow-up MRI scans showed resolution of the subdural changes. Subdural changes should be considered as one of the possible causes of unexpected symptoms in patients following lumbar spinal surgery.

Key Words: Lumbar spine, Subdural change, Magentic resonance imaging, Post-operative period

\section{Introduction}

Although magnetic resonance imaging (MRI) is frequently used to assess the lumbar spine, there are few reports in the medical literature that have evaluated using MRI immediately following spinal surgery [1-3]. Furthermore, descriptions of the subdural changes after lumbar spine surgery are also infrequent [2-4]. We recently encountered two cases with subdural change seen on MRI immediately after lumbar surgery. In both cases, the lesions spontaneously resolved on the follow-up MRI after one month. The purpose of this study is to report the subdural changes seen on MRI after lumbar spine surgery.

\section{Case Reports}

\section{Case 1}

A 48-year-old woman was admitted to our hospital with symptoms of pain and palsy in both legs due to lumbar degenerative spondylolisthesis of L4 (Meyerding grade 1). The straight leg raising test (SLRT) was positive at $70^{\circ}$ in the right side and at $40^{\circ}$ in the left side. Weakness of the left tibialis anterior and left extensor hallucis longus muscles was noted with 4/5 manual muscle test (MMT) strength. The patient had undergone transvaginal hysterectomy due to a myoma one year previously. She had no history of any other diseases or coagulation abnormalities. No

Received Sep 15, 2010; 1st Revised Oct 22, 2010; 2nd Revised Nov 3, 2010; Accepted Nov 4, 2010

Corresponding author: Toshinori Sakai, MD, PhD

Department of Orthopedics, Institute of Health Biosciences, The University of Tokushima Graduate School,

3-18-15 Kuramoto-cho, Tokushima 770-8503, Japan

Tel: +81-88-633-7240, Fax: +81-88-633-0178, E-mail: norinoridowluck@yahoo.co.jp 
coagulation abnormalities were found on the preoperative examination. The platelet count was $26.4 \times 10^{4} / \mu$, the prothrombin time (PT) was 10.5 seconds, the PT-international normalized ratio (PT-INR) was 0.9 and the activated partial thromboplastin time (APTT) was 32.7 seconds.

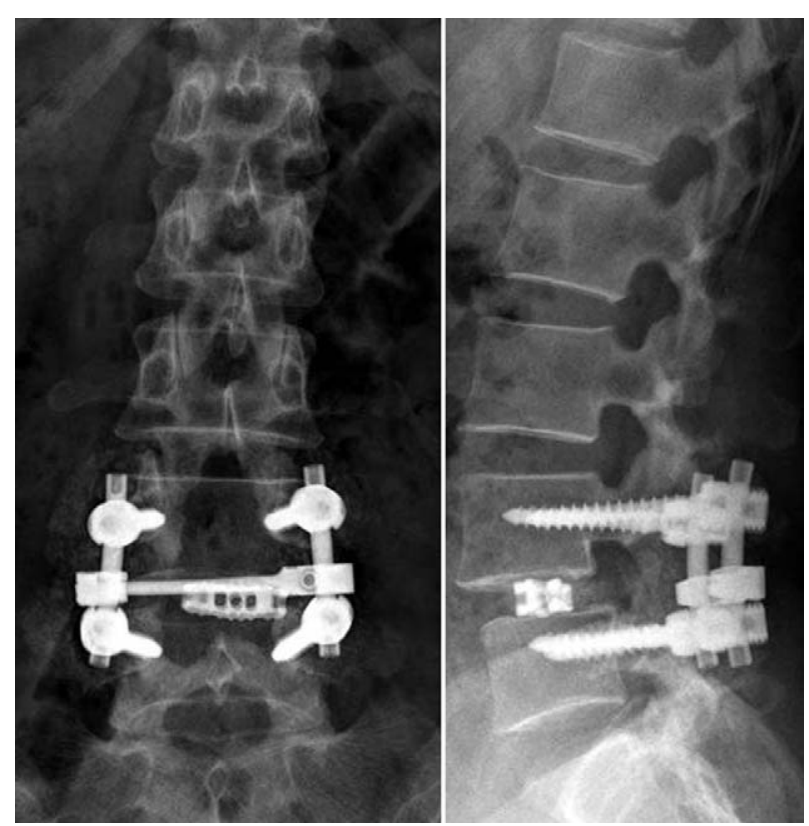

Fig. 1. The postoperative plain X-ray films show a successful L4 wide laminectomy and transforaminal lumbar interbody fusion of L4-L5 in a patient (case 1) with lumbar degenerative spondylolisthesis.
The patient underwent a wide L4 laminectomy and transforaminal lumbar interbody fusion of L4-L5 for the lumbar degenerative spondylolisthesis and spinal stenosis (Fig. 1). The operative time was 254 minutes and the intraoperative blood loss was $490 \mathrm{ml}$. Intraoperatively, there were no complications, including dural laceration. Two drains were placed in the surgical site to prevent the occurrence of an epidural hematoma. Immediately after the operation, both the pain and palsy of the patient's legs were improved. The SLRT bilaterally improved to $80^{\circ}$, and the muscle strength started to increase. Cerebrospinal fluid (CSF) leakage was not found postoperatively in the drain tubes. A nonsteroidal anti-inflammatory drug (NSAID) was prescribed for postoperative pain management. The drain tubes were removed two days after the operation. The patient was gradually mobilized using a walker and a lumbar corset. She gradually began to feel an abnormal sensation around her buttock. The feeling gradually increased and particularly when she began mobilizing.

Seven days after the operation, MRI revealed complete decompression of the dural sac, and there was a lesion within the subdural compartment from the L4 through the S2 level. The lesion occupied the subdural space and it was iso-intense to the spinal cord on the T1-weighted images and hyper-intense on the T2-weighted sequences (Fig. 2), and these findings were suggestive of a hematoma. Two

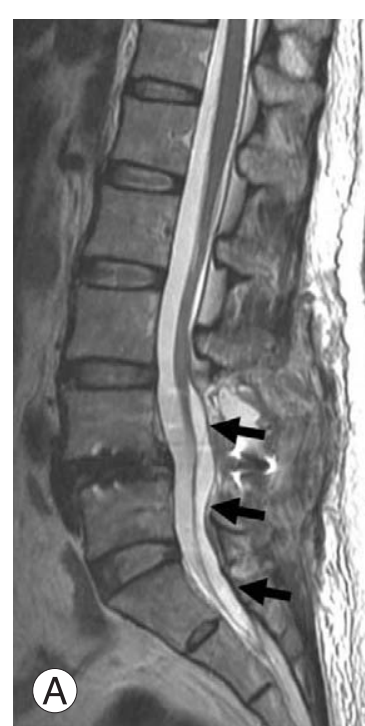

T2-WI

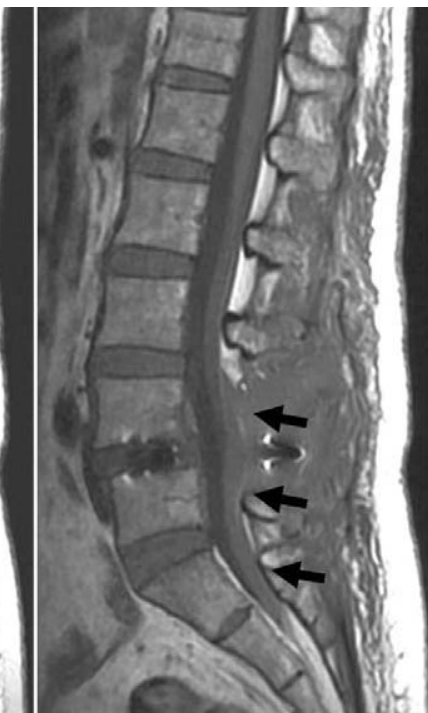

$\mathrm{T} 1-\mathrm{Wl}$

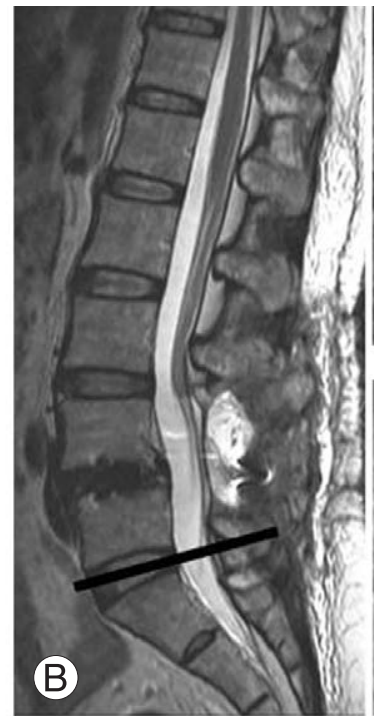

B

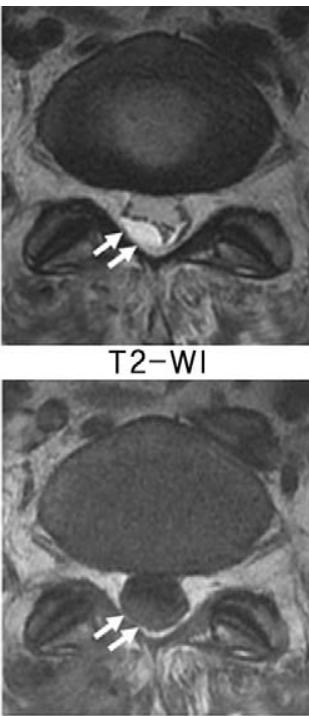

$\mathrm{T} 1-\mathrm{WI}$

Fig. 2. (A) The sagittal slice magnetic resonance imaging (MRI) images obtained seven days after the operation. The images revealed a lesion within the subdural compartment at the L4-S2 level that was iso-intense to the spinal cord on the T1-weighted images and hyper-intense on the T2-weighted images. (B) The axial slice MRI images through the L5-S1 disc level shows a biconvex subdural hematoma on the right dorsal side of the dura. Especially note that this is not the surgical site. 
weeks later, her symptoms began to gradually improve without intervention. Follow-up MRI obtained 25 days after the operation revealed resolution of the subdural lesion and a morphologically normal cauda equina (Fig. 3).

\section{Case 2}

A 76-year-old woman was admitted to our hospital with symptoms of left leg pain and intermittent claudication due to lumbar isthmic spondylolisthesis of L5. Preoperatively, she had no muscle weakness in her legs. Her past medical history included hypertension, which was well-controlled with medication, and hepatitis C. On her preoperative examination, the platelet count was $12.9 \times 10^{4} / \mu \mathrm{l}$, the PT was 10.6 seconds, the PT-INR was 0.9 and the APTT was 33.6 seconds.

We performed a wide L5 laminectomy and posterolateral fusion of L5-S1 for the lumbar isthmic spondylolisthesis. The operative time was 240 minutes and intraoperative blood loss was $460 \mathrm{ml}$. There were no intraoperative com-

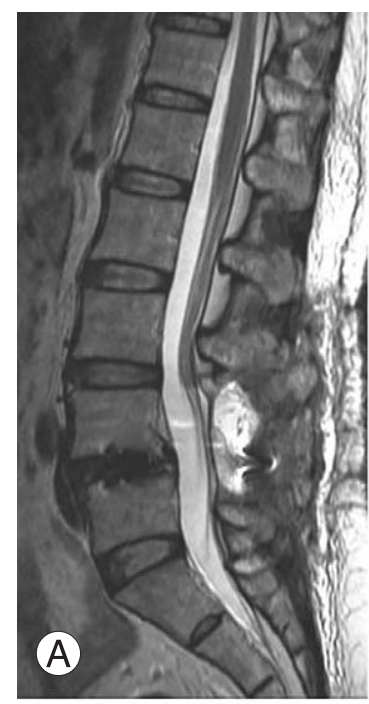

$\mathrm{T} 2-\mathrm{WI}$

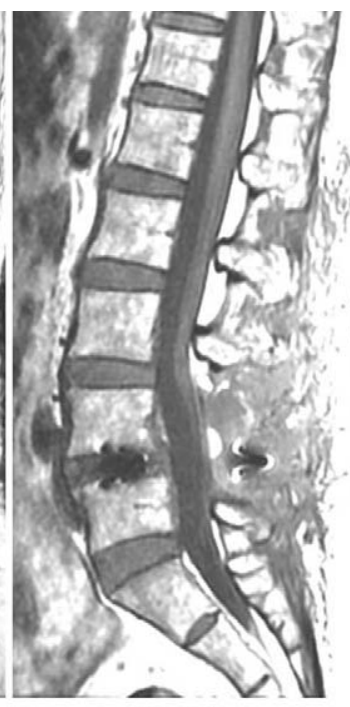

T1-WI
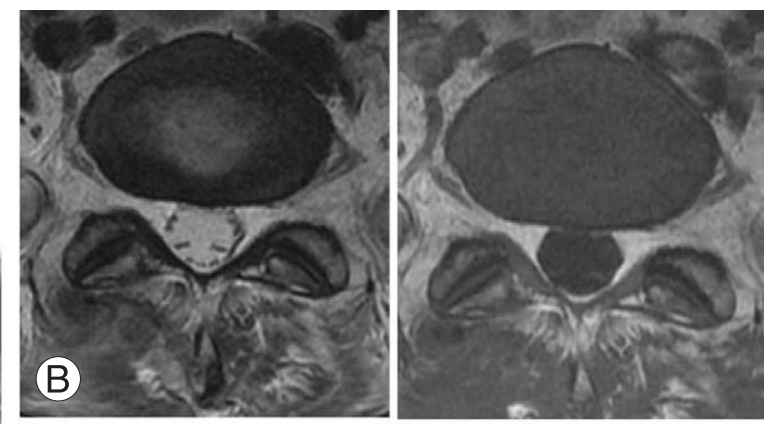

Fig. 3. The follow-up magnetic resonance imaging obtained 25 days after the operation show the resolution of the subdural lesion (A) and a morphologically normal cauda equina (B).

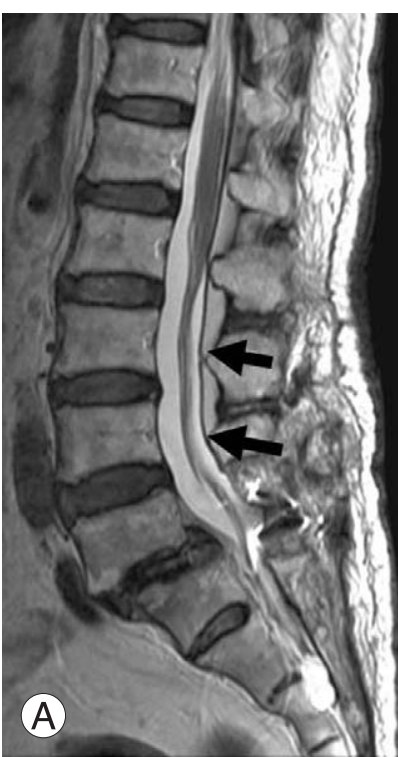

T2-WI

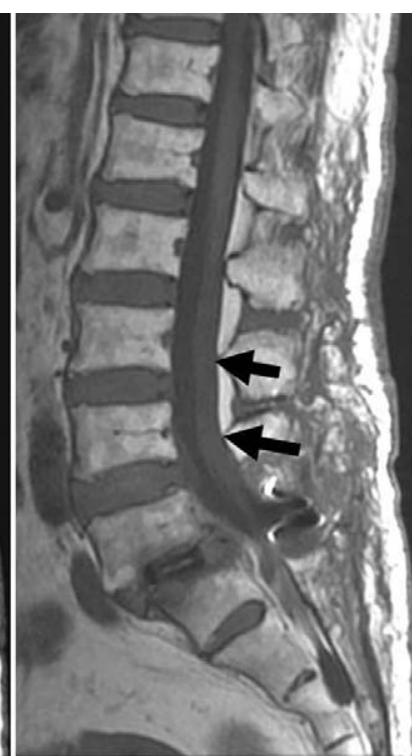

T1-WI

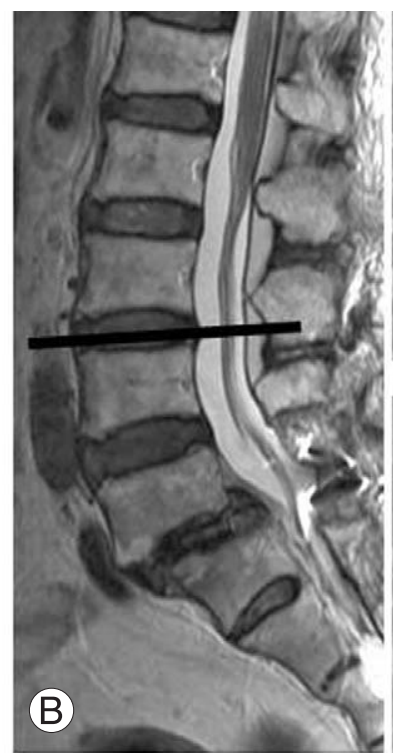

B

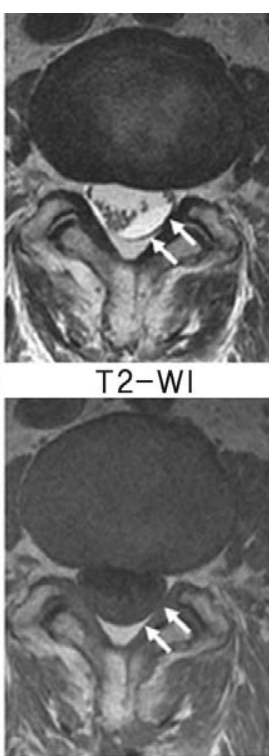

$\mathrm{T} 1-\mathrm{WI}$

Fig. 4. (A) The sagittal slice magnetic resonance imaging (MRI) images obtained 7 days after posterolateral lumbar fixation of L5-S1 show a lesion within the subdural compartment at the L2-S2 level. The lesion is iso-intense to the spinal cord on the T1-weighted images and hyper-intense on the T2-weighted images (Case 2). (B) The axial slice MRI images through the L3-L4 disc level show a biconvex subdural hematoma on the left dorsal side of the dura. Especially note that this is not the surgical site. 
plications. We inserted two drains in the surgical site to prevent the occurrence of an epidural hematoma. The patient's left leg pain resolved immediately after the operation. CSF leakage was not found in the drain tubes postoperatively. A NSAID was prescribed for the postoperative pain management. Two days after the operation, the drain tubes were removed and she was mobilized while wearing a lumbar corset. Then she noticed muscle weakness of the left leg that diffusely involved the iliopsoas, quadriceps, tibialis anterior and the left extensor hallucis longus, and each of these muscle groups' strength was 4/5 on MMT.

Seven days after the operation, MRI revealed total decompression of the dural sac, and there was a lesion within the subdural compartment from L2 through S2. The lesion occupied the subdural space, it was iso-intense to the spinal cord on the T1-weighted images and it was hyperintense on the T2-weighted sequences (Fig. 4), which was all compatible with a subdural hematoma. Two weeks later, her muscle strength gradually increased without intervention and it subsequently returned to normal. The follow-up MRI obtained 29 days after the operation showed resolution of the subdural lesion and a morphologically normal cauda equina (Fig. 5).

\section{Discussion}

There are few reports describing the post-operative MRI findings after lumbar surgery, with several studies having evaluated postoperative lumbar epidural hematomas [1-3]. Yet few reports have describe the subdural changes after lumbar spine surgery [4-6]. Matsui et al. [4] examined the time-related changes seen on postoperative MRI scans after lumbar laminectomy in 10 patients and they reported that cauda equina adhesions and shrinkage of the thecal sac were closely correlated with laminectomy. Their group also evaluated the vascular permeability changes of the cauda equina after lumbar laminectomy in rats and they speculated that accelerated permeability may enhance cauda equina adhesions [5]. But this mechanism has not yet been clinically clarified. Furthermore, these cauda equina adhesions and the thecal sac shrinkage cannot account for the subdural MRI changes seen in our study.

We hypothesize that the subdural lesion was likely a spinal subdural hematoma. We assume that some operative procedures may have affected the subdural or intradural vessels. Fortunately, the present cases had only mild symptoms, and their symptoms subsided and the lesions resolved spontaneously. We did not perform a pathological examination, and hence we could not verify the lesions. In the published literature, there is one report of a 36-year-old man who presented six weeks after surgery with incapacitating pain and he was found to have a subdural, but extra-arachnoid, fluid collectionn [6].

MRI is generally thought to be the best tool to assess the subdural space in the lumbar spine. In the presented cases, the subdural changes demonstrated slightly higher-signal intensity than CSF on the T1-weighted images. However, on the T2-weighted images, the subdural lesions had the same signal intensity as that of the CSF. The axial views can reveal the intradural location of the changes. The subdural change can be ventral or dorsal to the spinal cord or cauda equina and it has a variable shape, including a cres-

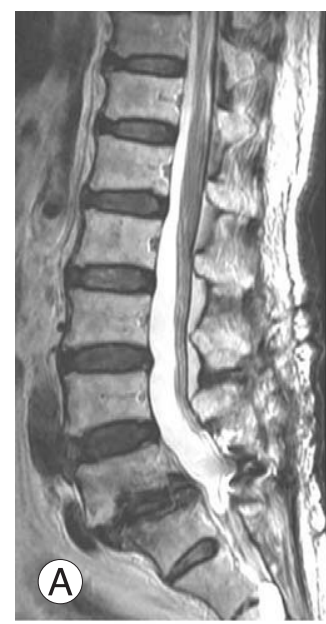

T2-WI

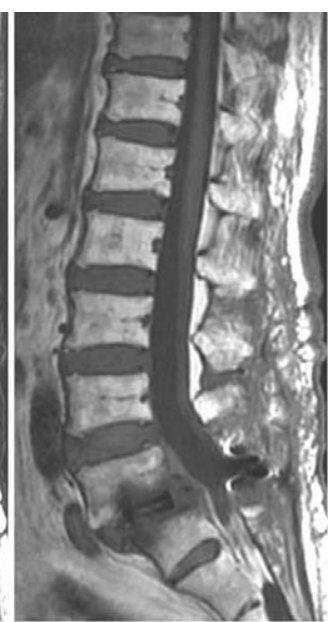

$\mathrm{T} 1-\mathrm{WI}$

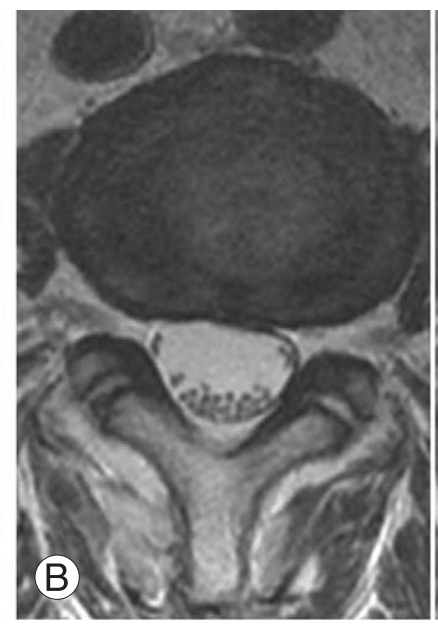

$\mathrm{T} 2-\mathrm{WI}$

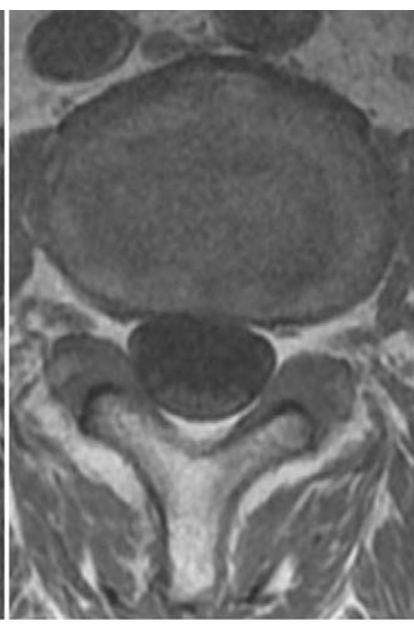

$\mathrm{T} 1-\mathrm{WI}$

Fig. 5. (A, B) The follow-up magnetic resonance imaging obtained 29 days after the operation shows resolution of the subdural lesion and a morphologically normal cauda equina. 
cent, biconvex, circumferential or mixture of shapes at different levels.

In this paper, we have described two patients with subdural post-operative MRI changes following lumbar spinal surgery. These changes likely represented subdural hematomas, and they may have been associated with postoperative symptoms. In our cases, both the patients had mild symptoms that resolved spontaneously and the followup MRI scans showed resolution of the subdural changes. For patients with unexpected symptoms following lumbar spinal surgery, subdural changes should be considered as one of the possible causes of the symptoms.

\section{REFERENCES}

1. Djukic S, Vahlensieck M, Resendes M, Genant HK. The lumbar spine: postoperative magnetic resonance imaging. Bildgebung 1992;59:136-46.
2. Ross JS. Magnetic resonance imaging of the postoperative spine. Semin Musculoskelet Radiol 2000;4:281-91.

3. Sokolowski MJ, Garvey TA, Perl J 2nd, et al. Prospective study of postoperative lumbar epidural hematoma: incidence and risk factors. Spine (Phila Pa 1976) 2008;33:10813.

4. Matsui H, Tsuji H, Kanamori M, Kawaguchi Y, Yudoh K, Futatsuya R. Laminectomy-induced arachnoradiculitis: a postoperative serial MRI study. Neuroradiology 1995;37:660-6.

5. Nakano M, Matsui H, Miaki K, Yamagami T, Tsuji H. Postlaminectomy adhesion of the cauda equina: changes of postoperative vascular permeability of the equina in rats. Spine (Phila Pa 1976) 1997;22:1105-14.

6. Reinsel TE, Goldberg E, Granato DB, Wilkinson S, Penn R. Spinal subdural hematoma: a rare cause of recurrent postoperative radiculopathy. J Spinal Disord 1993;6:62-7. 